\title{
Anomia training and brain stimulation in chronic aphasia
}

\author{
Maria Cotelli' ${ }^{1}$, Anna Fertonani' ${ }^{1}$, Antonio Miozzo², \\ Sandra Rosini ${ }^{1}$, Rosa Manenti ${ }^{1}$, Alessandro Padovani ${ }^{2}$, \\ Ana Ines Ansaldo ${ }^{3}$, Stefano F. Cappa ${ }^{4}$, \\ and Carlo Miniussi ${ }^{1,5}$ \\ ${ }^{1}$ IRCCS Centro San Giovanni di Dio Fatebenefratelli, Brescia, Italy \\ ${ }^{2}$ Centre for Brain Aging and Neurodegenerative Disorders, Neurology Unit, \\ University of Brescia, Italy \\ ${ }^{3}$ Département D’orthophonie et Audiologie, Faculté de Médecine, \\ Université de Montréal; Centre de Recherche de l'Institut Universitaire de \\ Gériatrie de Montréal, Canada \\ ${ }^{4}$ Vita-Salute University and San Raffaele Scientific Institute, Milan, Italy \\ ${ }^{5}$ Department of Biomedical Sciences and Biotechnologies, National \\ Neuroscience Institute, University of Brescia, Brescia, Italy
}

\begin{abstract}
Recent studies have reported enhanced performance on language tasks induced by non-invasive brain stimulation, i.e., repetitive transcranial magnetic stimulation (rTMS), or transcranial direct current stimulation (tDCS), in patients with aphasia due to stroke or Alzheimer's disease (AD). The first part of this article reviews brain stimulation studies related to language recovery in aphasic patients. The second part reports results from a pilot study with three chronic stroke patients who had non-fluent aphasia, where real or placebo rTMS was immediately followed by 25 minutes of individualised speech therapy. Real rTMS consisted of high-frequency rTMS over the left dorsolateral prefrontal cortex (BA 8/9) for 25 minutes. Each patient underwent a total of four weeks of intervention. P1 underwent four weeks of real rTMS
\end{abstract}

Correspondence should be addressed to Maria Cotelli, PhD, IRCCS Centro San Giovanni di Dio Fatebenefratelli, Via Pilastroni, 4, 25125 Brescia, Italy. E-mail: mcotelli@ fatebenefratelli.it

This work was supported by James S. McDonnell Foundation and Associazione Fatebenefratelli per la Ricerca.

(C) 2011 Psychology Press, an imprint of the Taylor \& Francis Group, an Informa business http://www.psypress.com/neurorehab http://dx.doi.org/10.1080/09602011.2011.621275 
(5 days/week) where individualised speech therapy was provided for 25 minutes immediately following each rTMS session. P2 and P3 each underwent two weeks of placebo rTMS, followed immediately by individualised speech therapy; then two weeks of real rTMS, followed immediately by individualised speech therapy. Assessments took place at 2, 4, 12, 24 and 48 weeks post-entry/baseline testing. Relative to entry/baseline testing, a significant improvement in object naming was observed at all testing times, from two weeks post-intervention in real rTMS plus speech therapy, or placebo rTMS plus speech therapy. Our findings suggest beneficial effects of targeted behavioural training in combination with brain stimulation in chronic aphasic patients. However, further work is required in order to verify whether optimal combination parameters (rTMS alone or speech therapy alone) and length of rTMS treatment may be found.

Keywords: TMS; rTMS; Naming; Speech therapy.

\section{INTRODUCTION}

Aphasia is a frequent consequence of stroke (Engelter, 2006; Laska, Hellblom, Murray, Kahan, \& Von Arbin, 2001) and is associated with increased mortality, decreased rates of functional recovery, and reduced probability of returning to work (Black-Schaffer \& Osberg, 1990; Paolucci et al., 1998; Tilling et al., 2001). While there is a general consensus that aphasia rehabilitation is effective (Berthier \& Pulvermuller, 2011; Cappa et al., 2003, 2005; Galletta, Rao, \& Barrett, 2011), high-quality clinical trials are lacking and there is insufficient evidence indicating which is the best approach to delivering speech and language therapy (Kelly, Brady, \& Enderby, 2010). Moreover, recent studies have shown that a high intensity of treatment is crucial for efficacy (Bhogal, Teasell, \& Speechley, 2003); however, from a clinical prospective, highly intensive treatments are often impractical.

Within this context, there is increasing interest in the potential enhancement of performance with non-invasive brain stimulation, i.e., repetitive transcranial magnetic stimulation (rTMS) or transcranial direct current stimulation (tDCS), applied to specific cortical areas (Miniussi et al., 2008). Facilitation effects have been observed in patients with stroke and dementia when performing a variety of cognitive tasks. These effects have been related to TMS-induced changes in cortical excitability resulting in functional reorganisation and improved cognitive performance. Specifically, the potential of rTMS or tDCS to trigger adaptive neuroplasticity in neurological patients has been related to three main mechanisms: (1) the reactivation of canonical networks, partly damaged or made dysfunctional by the cerebral lesion; (2) the recruitment of compensatory networks, mostly contralateral homologue cortical regions; and (3) the additional recruitment of perilesional 
sub-optimally functioning areas (see Miniussi \& Rossini, this issue, for a discussion). However, the mechanisms responsible for rTMS- and tDCS-induced changes in neural activity remain largely unknown. There is, however, evidence that rTMS and tDCS techniques can modify neuronal excitation through different mechanisms: TMS elicits action potentials in neurons, whereas tDCS does not (see Paulus, this issue). However, tDCS effectively modifies both the evoked cortical response to afferent stimulation, and the post-synaptic activity level of cortical neurons, presumably by inducing a shift in intrinsic neuronal excitability (Bindman, Lippold, \& Redfearn, 1962). In other words, both techniques can modify cortical plasticity by increasing excitability in cortical neurons within a specific network and, in doing so, improve the cognitive ability sustained by the stimulated network.

Specifically, regarding rTMS, there is evidence that the frequency of stimulation modulates neural activity. High-frequency rTMS $(\geq 5 \mathrm{~Hz})$, has been shown to increase cortical excitability, whereas low-frequency rTMS $(\leq 1 \mathrm{~Hz})$ can inhibit "maladaptive" plasticity, which prevents recovery from aphasia (Martin, Naeser, Ho, Doron, et al., 2009). The administration of rTMS to the anterior portion of the right homologue of Broca's area (pars triangularis) improves picture naming in patients with non-fluent aphasia (Barwood, Murdoch, Whelan, Lloyd, Riek, O'Sullivan, Coulthard, \& Wong, 2011; Barwood, Murdoch, Whelan, Lloyd, Riek, O’Sullivan, Coulthard, Wong, et al., 2011; Hamilton et al., 2010; Naeser, Martin, Lundgren, et al., 2010; Naeser, Martin, Nicholas, Baker, Seekins, Helm-Estabrooks, et al., 2005). In a stroke patient with chronic non-fluent aphasia, the same authors reported improvement of language following treatment with continuous positive airway pressure (CPAP) for sleep apnoea, as well as following CPAP plus slow-rTMS, to suppress pars triangularis (Naeser, Martin, Lundgren, et al., 2010). Naeser et al. (Naeser, Martin, Nicholas, Baker, Seekins, Kobayashi, 2010) argue that low-frequency rTMS over the right pars triangularis suppresses maladaptive right hemisphere frontal activations, and thus allows for the activation of left hemispheric perilesional and perisylvian areas, as well as the left supplementary motor area, which support recovery in non-fluent aphasic patients. However, in the study by Martin and co-authors (Martin, Naeser, Ho, Doron, et al., 2009), only one of two patients with chronic non-fluent aphasia showed language improvement following low-frequency rTMS on the right pars triangularis. Martin et al. (Martin, Naeser, Ho, Doron, et al., 2009) argue that the shift to left hemisphere activation post-rTMS observed in the fMRI scans of the good responders supports the idea that restoration of the left hemisphere language network is linked, at least in part, to a better recovery of naming in non-fluent aphasia. Moreover, Martin, Naeser, Ho, Treglia, et al. (2009) reported improved naming performance following a combined behavioural and rTMS treatment in a pilot single-case study, in which rTMS stimulation was immediately followed by constraint-induced language 
therapy. These pilot data suggest that a combined behavioural-rTMS treatment could be more efficient than behavioural treatment alone.

In this vein, Kakuda, Abo, Kaito, et al. (2010) demonstrated that the application of low-frequency rTMS to an area that is homologous to the most activated area, as evaluated in a pre-treatment fMRI acquisition, resulted in improvement of language abilities in three chronic stroke patients. In a subsequent study, the authors applied rTMS over the left Wernicke's area combined with language therapy in two post-stroke patients with "sensorydominant" aphasia (Kakuda, Abo, Uruma, et al., 2010). Ten sessions of treatment were delivered during a 6-day hospitalisation period, followed by a weekly outpatient rTMS treatment for 3 months. The study showed an improvement of comprehension abilities in both patients at the end of the 6th day, maintained at the 3-month post-discharge period.

A recent randomised, controlled, blinded pilot study has investigated the effect of low-frequency rTMS over the right-hemispheric pars triangularis portion of the Broca's area homologue in 10 left-brain-damaged patients with post-stroke aphasia (Weiduschat et al., 2011). Patients received, in addition to conventional speech and language therapy, multiple sessions of rTMS. Using positron emission tomography (PET) this study revealed a shift of activation towards the right hemisphere in the placebo group, but not in the real rTMS group of patients. Furthermore, only patients in the real rTMS group improved their performance in the Aachener Aphasia Test (AAT) global score. The authors suggest that low-frequency rTMS applied on the right-hemispheric pars triangularis portion of the Broca's area homologue prevents right-hemispheric lateralisation resulting in a better clinical improvement.

Furthermore, Szaflarski and co-authors (2011) provide preliminary evidence regarding the safety and efficacy of fMRI-guided high-frequency rTMS (intermittent theta burst stimulation) applied to the residual left hemispheric Broca's area in eight left-brain-damaged patients with post-stroke aphasia. The study showed an improvement in semantic fluency after 2 weeks of stimulation that was associated with a significant shift of the fMRI signal to the affected hemisphere. Finally, Jung and co-workers (2010) report a significant improvement of naming and comprehension performances in a post-stroke patient affected by crossed aphasia, following the application of low-frequency rTMS over the left parietal cortex.

tDCS is another way of promoting neuroplasticity to enhance cognitive performance. In tDCS, a weak electrical current is directly applied to the head to generate an electrical field that modulates neuronal activity (see Paulus, this issue). Anodal tDCS (atDCS) has a general facilitation effect and causes membrane depolarisation, whereas cathodal tDCS (ctDCS) has a general inhibitory effect and causes membrane hyperpolarisation (cathodal stimulation, see Liebetanz, Nitsche, Tergau, \& Paulus, 2002). Behavioural 
facilitatory tDCS effects have been highlighted (see Vallar \& Bolognini, this issue) with respect to implicit motor learning (Nitsche et al., 2003; Reis et al., 2009), working memory (Fregni et al., 2005; Ohn et al., 2008), pitch memory (Vines, Schnider, \& Schlaug, 2006), perception (Antal et al., 2004), and language (Fertonani, Rosini, Cotelli, Rossini, \& Miniussi, 2010; Iyer et al., 2005; Sparing, Dafotakis, Meister, Thirugnanasambandam, \& Fink, 2008). Interestingly, and particularly relevant to the neurorehabilitation field, these tDCS-induced modifications of cortical excitability and behaviour can outlast the stimulation period itself. Hence, there is growing interest in applying these methodologies therapeutically, in order to potentiate the effects of cognitive rehabilitation, and to reduce cognitive deficits in patients with chronic and neurodegenerative diseases.

Monti and co-authors (2008) reported naming facilitation following cathodal stimulation over the damaged left frontotemporal areas in eight patients with chronic, non-fluent, post-stroke aphasia. Baker, Rorden, and Fridriksson (2010) performed a combined behavioural tDCS study with left-braindamaged patients receiving 5 days of computerised anomia therapy concurrently with tDCS over the damaged left hemisphere or sham stimulation. This study revealed significantly improved naming accuracy in patients treated with anodal tDCS compared with the sham tDCS patients. In addition, the treatment effect persisted for at least 1 week after treatment. Fridriksson et al. (2011) applied the same procedure used by Baker et al. (2010) in eight left-brain-damaged patients with chronic fluent aphasia, and showed that anodal tDCS administered during language treatment decreased verbal reaction times during naming, as assessed immediately post-treatment and 3 weeks later. Another recent study (Fiori et al., 2011) highlights the beneficial effects of 5 days of anodal tDCS in three aphasic patients. The stimulation was applied to Wernicke's area while patients were executing a naming task. This procedure produced an improvement in naming accuracy that lasted for 3 weeks.

In a randomised, double-blind, sham controlled crossover trial, Floel and co-workers (2011) explored whether anodal tDCS compared to cathodal tDCS and placebo stimulation applied over the right temporo-parietal cortex would improve the success of anomia training in a group of 12 poststroke aphasic left-brain-damaged patients. This finding indicates that all treatment conditions led to a significant increase of naming ability, with a greater effect of anodal tDCS as compared to cathodal and placebo tDCS.

Kang and colleagues (2011) evaluated the hypothesis that cathodal tDCS applied on the right Broca's homologue could improve picture naming in patients with post-stroke aphasia. Patients received 5 consecutive days of cathodal tDCS followed or preceded (with a minimum interval of 1 week) by 5 days of placebo tDCS and simultaneous language therapy. Results demonstrated that cathodal tDCS applied over the right Broca's homologue 
combined with language therapy can improve picture naming task performance in post-stroke aphasic patients.

Furthermore, You, Kim, Chun, Jung, and Park (2011), in a prospective, double-blind, sham-controlled study assessed whether anodal tDCS delivered to the left superior temporal gyrus, or cathodal tDCS to the right superior temporal gyrus in comparison with sham tDCS could ameliorate aphasic symptoms. Cathodal tDCS over right superior temporal areas brought about significantly greater improvements in auditory verbal comprehension as compared to the other two conditions.

In summary, recent studies report enhanced cognitive performance following rTMS or tDCS, to specific cortical areas in a variety of patients with neurological diseases (see Table 1). Specifically, in chronic aphasia patients, non-invasive brain stimulation has been shown to increase the number of correct responses and to reduce response times. Moreover, recent studies suggest that these effects can persist over time (Baker et al., 2010; Barwood, Murdoch, Whelan, Lloyd, Riek, O'Sullivan, Coulthard, \& Wong, 2011; Fiori et al., 2011; Floel et al., 2011; Fridriksson et al., 2011; Hamilton et al., 2010; Kakuda, Abo, Kaito, et al., 2010; Kakuda, Abo, Uruma, et al., 2010b; Martin, Naeser, Ho, Doron, et al., 2009; Martin, Naeser, Ho, Treglia, et al., 2009; Naeser, Martin Lundgren, et al., 2010; Naeser, Martin Nicholas, Baker, Seekins, Helm-Estabrooks, et al., 2005; Naeser, Martin, Nicholas, Baker, Seekins, Kobayashi, et al., 2005; Naeser, Martin, Treglia, et al., 2010).

The present study reports the results of a combined rTMS anomia training approach administered to three patients with post-stroke chronic aphasia (PWAs).

\section{PARTICIPANTS}

Three patients with post-stroke chronic aphasia (PWAs) were recruited for the present study. Time after onset of aphasia varied from 1 year to 4.5 years. Two PWAs had suffered a left middle cerebral artery infarction, PWA 1 (P1) and PWA 3 (P3), whereas one PWA had a left capsulo-thalamic haemorrhage, PWA 2 (P2). P2 and P3 were right-handed, P1 was left-handed. A clinical assessment showed full awareness of the deficits in each of the three patients.

Each patient underwent a neurological assessment, complete neuropsychological assessment (see Table 2) and neuroimaging diagnostic procedures (see Figure 1). They all presented non-fluent speech but no verbal dyspraxia. The ability to understand single words was preserved. They could repeat and read single words but had a naming deficit.

Exclusion criteria included clinical evidence of depression, clinical signs of hearing or vision impairment, a past history of epilepsy, implanted metal objects, psychosis or major depression, alcohol abuse and drug addiction. 
BRAIN STIMULATION IN APHASIA

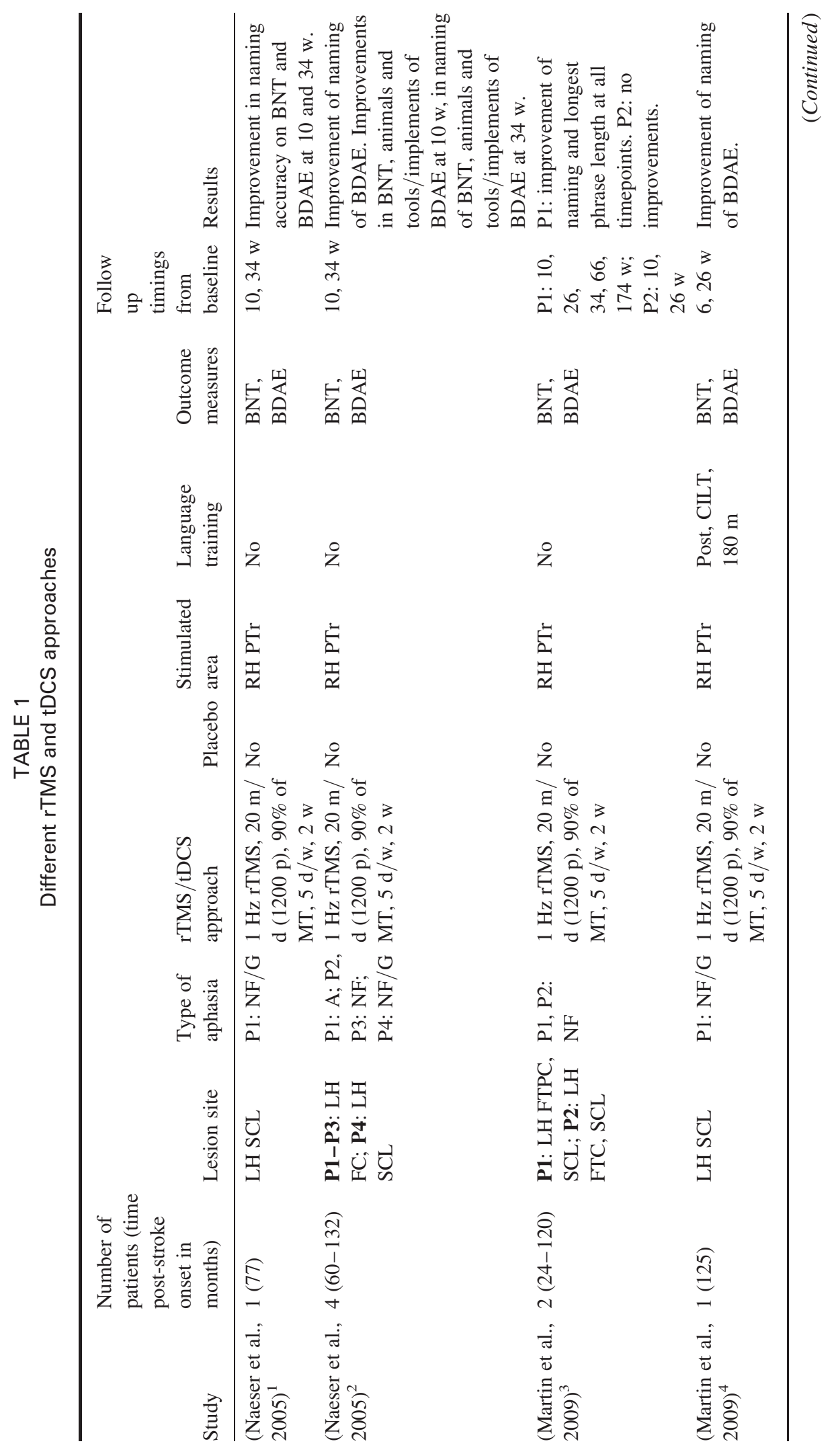


724 COTELLI ET AL.

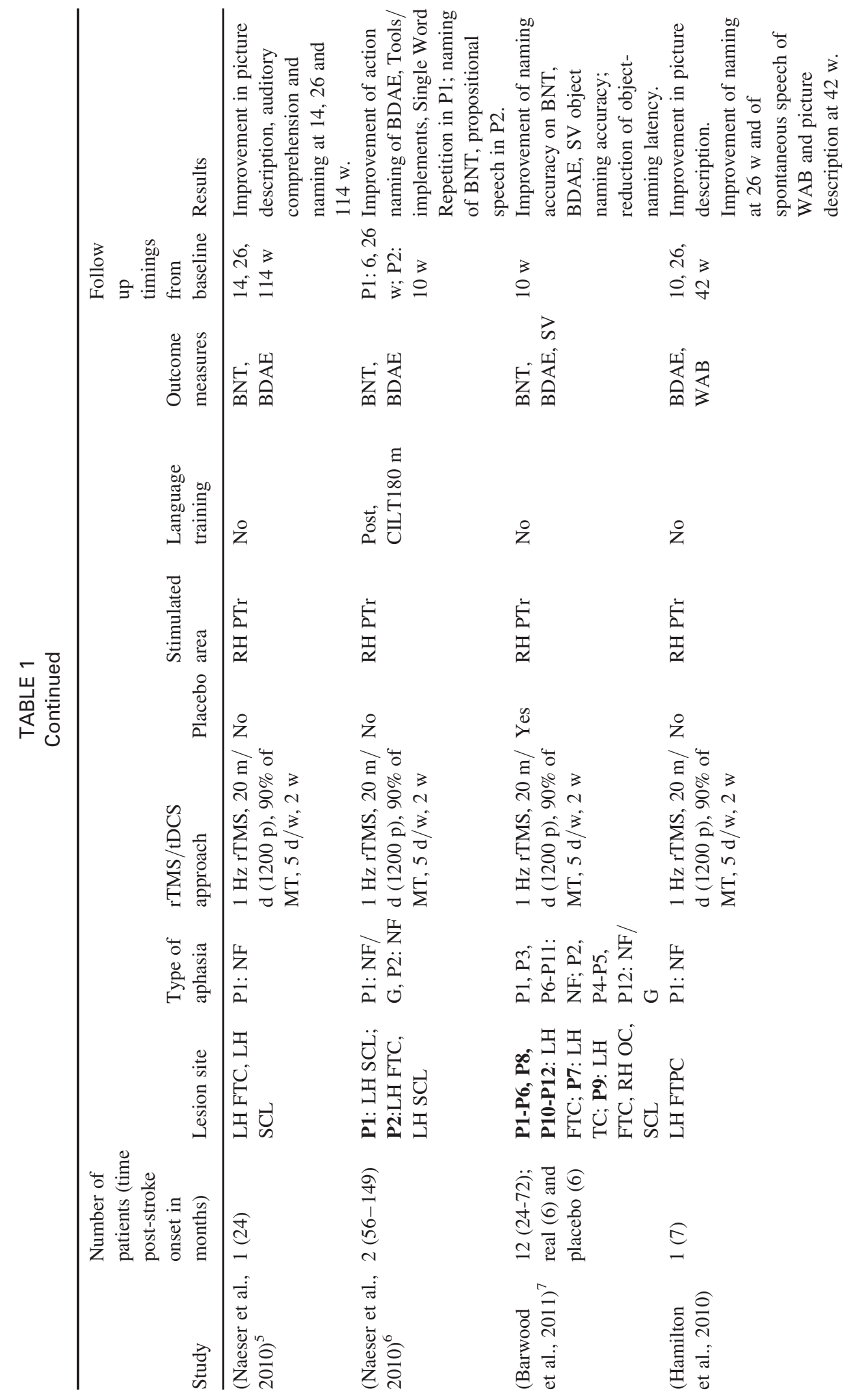



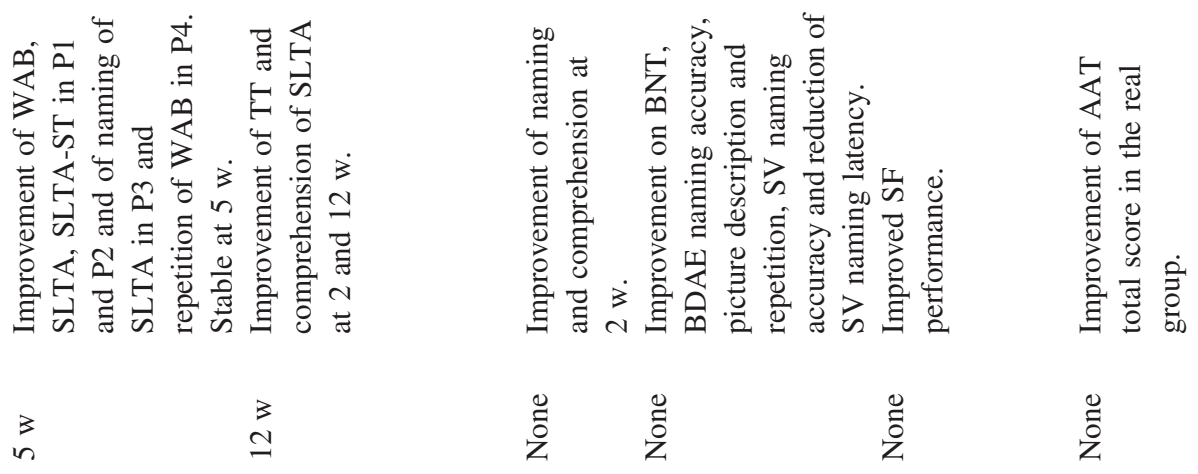

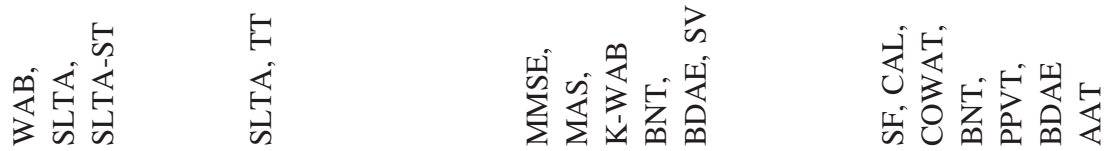
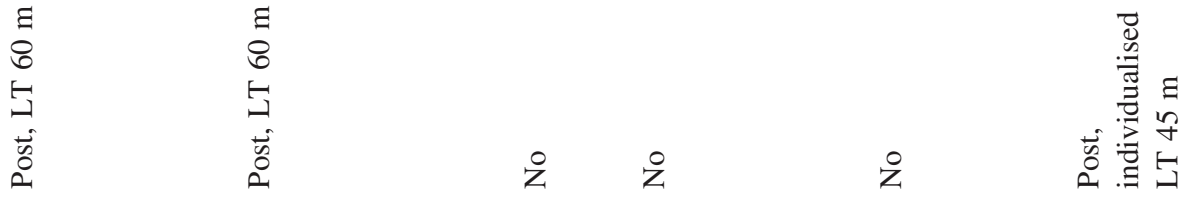

类

¿் உ்

式芒

疍

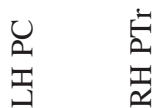

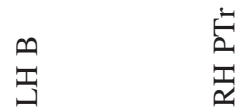

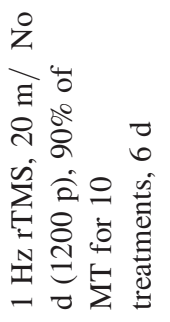

之े 之

之)

छ)

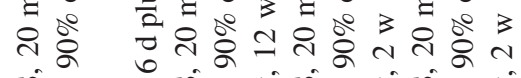

总的

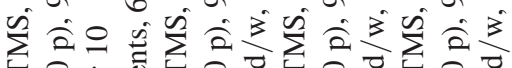

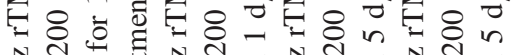

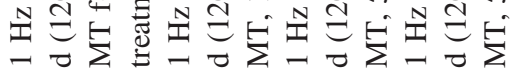

党苍

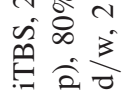

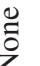

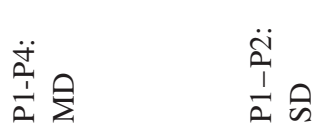

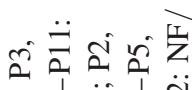

苂

छัँ

ํํำ

$\sum_{i=1}^{n} \hat{2} \hat{\Sigma}$

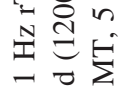

艺出罗

空

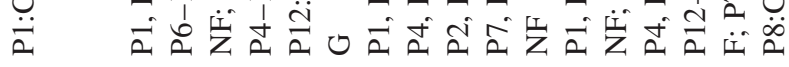

刍芒 导

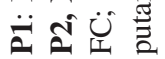

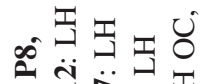

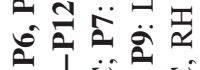

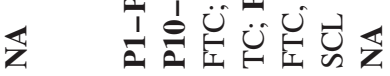

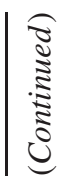

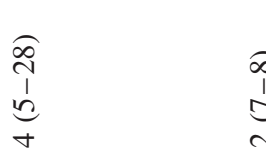

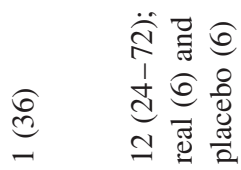

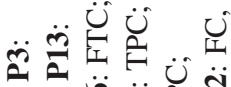

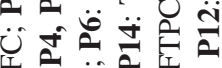

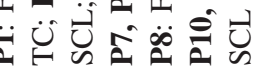

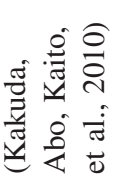

$\underset{N}{\infty}$

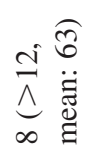

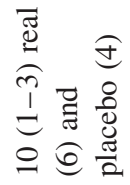

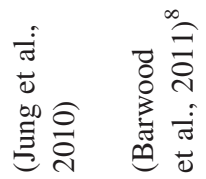

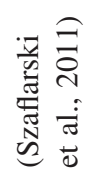

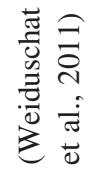


COTELLI ET AL.

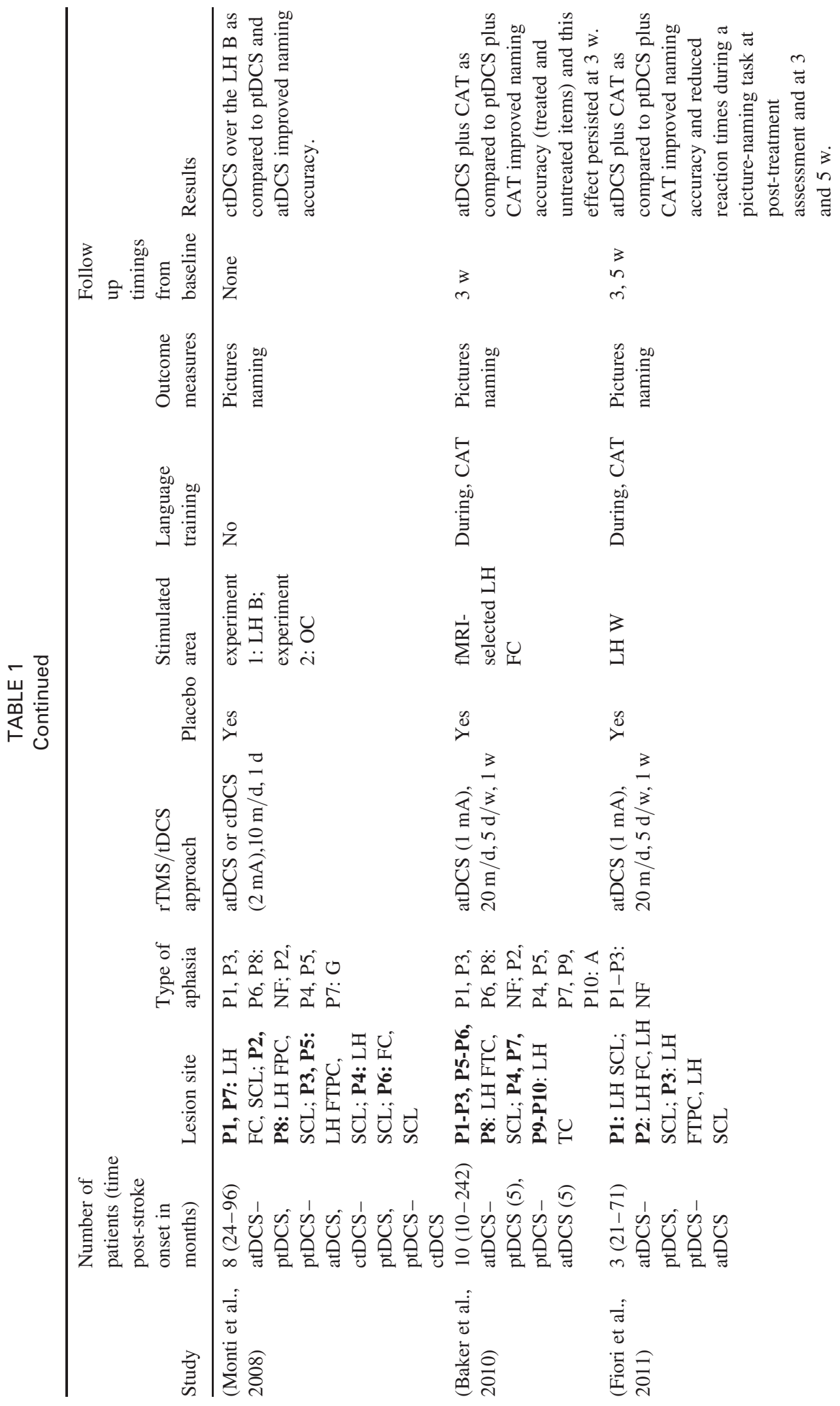



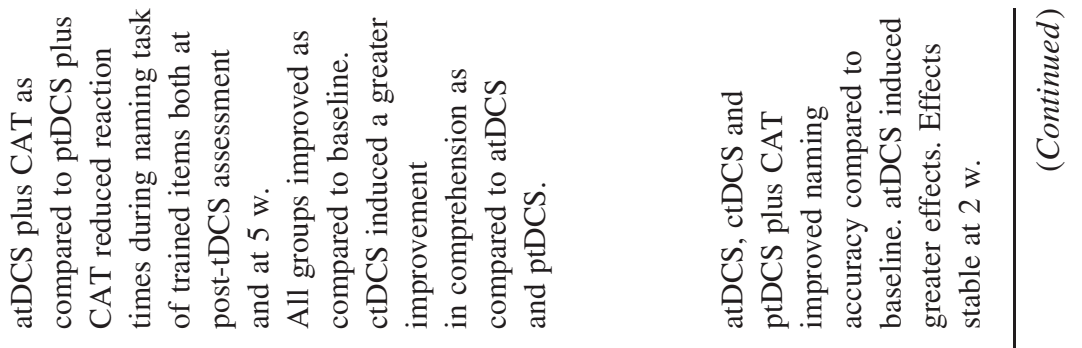

3

¿ั

$\stackrel{3}{4}$

总

$\sum_{\substack{1 \\ ⿱ 亠 乂}}^{\infty}$

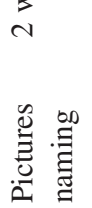

茯

导

ดิ

そ̊

害

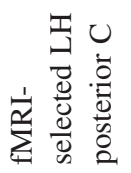

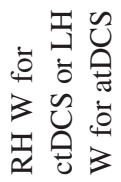

荘

$\stackrel{0}{\infty}$

$\overbrace{}^{\infty}$

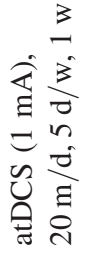

$>$

$\overbrace{\infty}^{\infty}$

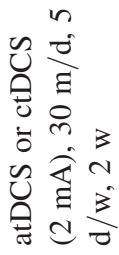

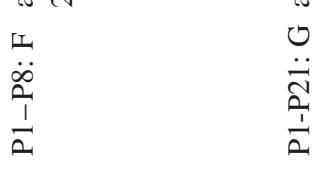

$\begin{array}{ll}\dot{\infty} & \ddot{\vec{\sigma}} \\ \frac{1}{a} & \frac{\dot{a}}{a}\end{array}$

$\begin{array}{ll}\dot{\infty} & \ddot{\bar{\Omega}} \\ \frac{1}{2} & \frac{\dot{a}}{2}\end{array}$

官

टृ ?

하우 3

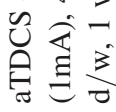

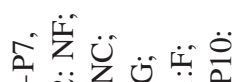

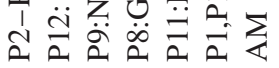

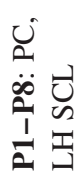

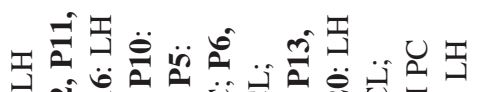

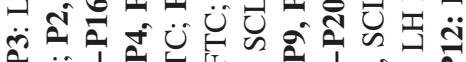

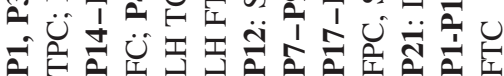

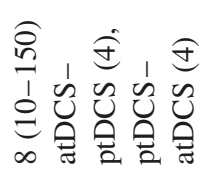

के $\dot{E} E$

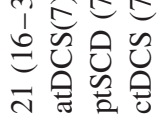

oิ

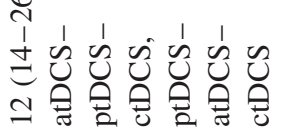

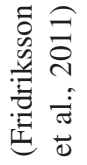

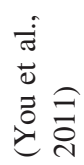

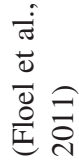


COTELLI ET AL.

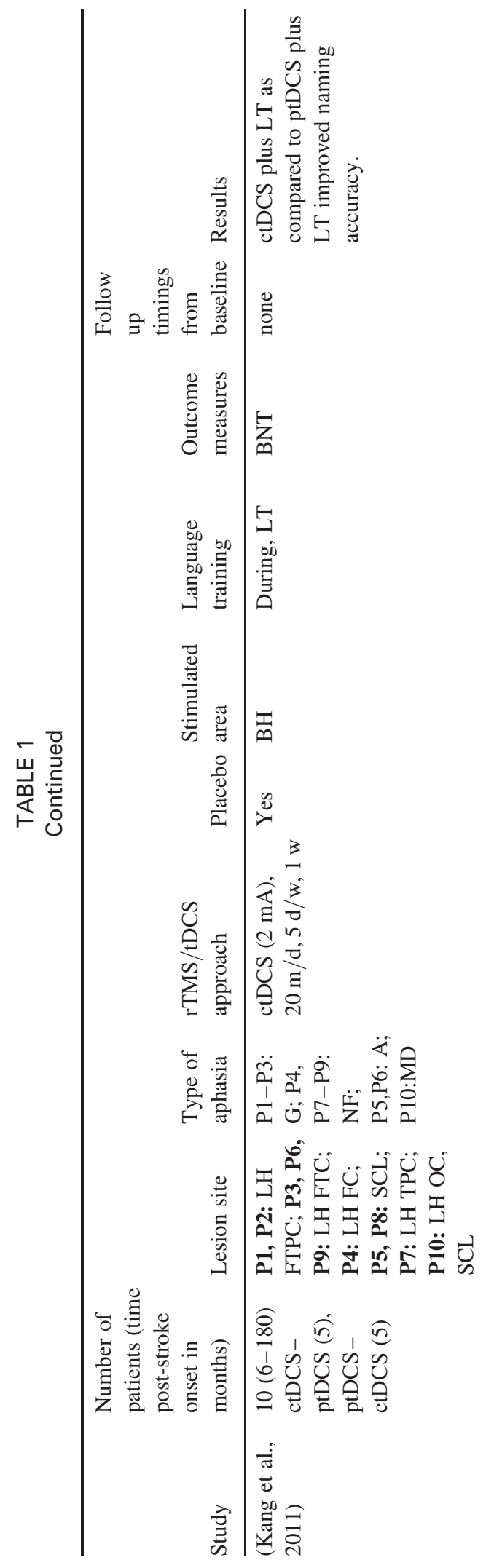

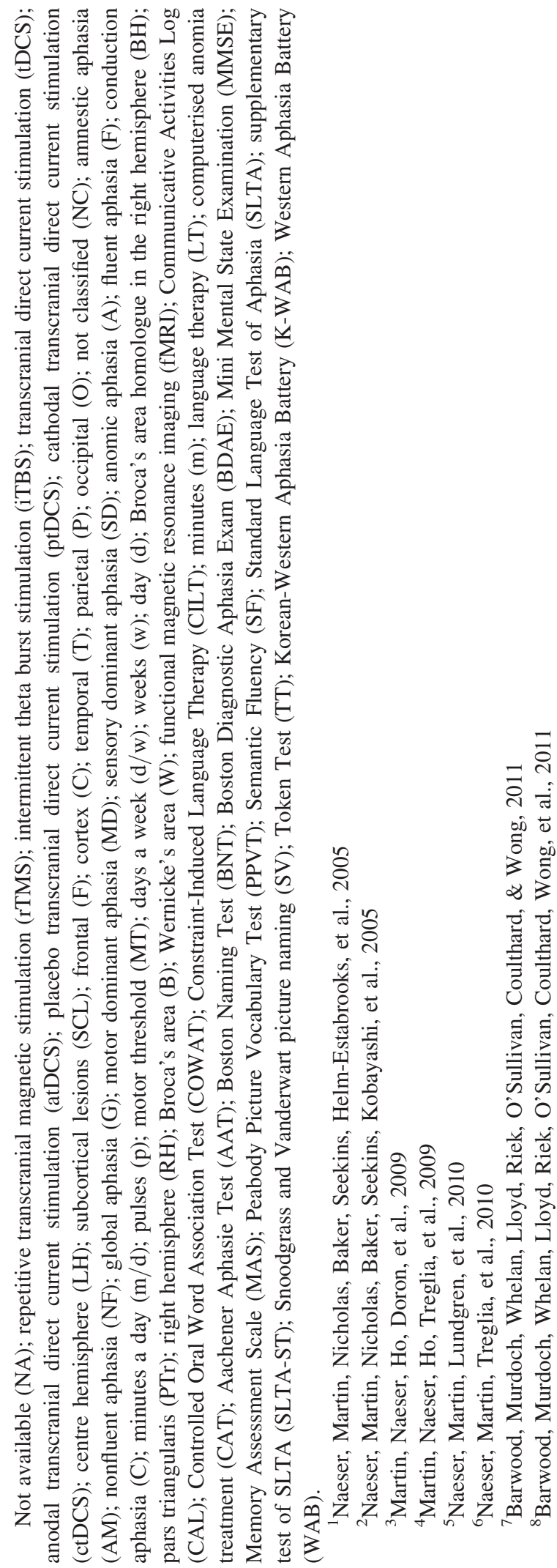


TABLE 2

Clinical features and baseline language assessment

\begin{tabular}{|c|c|c|c|}
\hline & P1 & $\mathrm{P} 2$ & P3 \\
\hline Age at entry into this study (years) & 41 & 45 & 71 \\
\hline Male/female & $\mathrm{F}$ & $\mathrm{M}$ & $\mathrm{M}$ \\
\hline Education (years) & 13 & 13 & 5 \\
\hline Aetiology (ischaemic/haemorrhagic) & I & $\mathrm{H}$ & I \\
\hline Time post onset (years) & 4.5 & 1 & 3.5 \\
\hline Length of a phrase (words) & 5 & 4 & 1 \\
\hline Neurological symptoms & Hemiparesis & Hemiplegia & Hemiparesis \\
\hline Handedness & Left & Right & Right \\
\hline \multicolumn{4}{|l|}{ Aachener Aphasie Test (AAT) } \\
\hline Token Test (Errors) & $19 / 50$ & $\mathbf{3 8} / \mathbf{5 0}$ & $\mathbf{2 7} / \mathbf{5 0}$ \\
\hline Repetition & $132 / 150$ & $106 / 150$ & 83/150 \\
\hline Writing & $86 / 90$ & $62 / 90$ & $8 / 90$ \\
\hline Naming & $105 / 120$ & $94 / 120$ & $13 / 120$ \\
\hline Comprehension & $109 / 120$ & $82 / 120$ & $42 / 120$ \\
\hline \multicolumn{4}{|c|}{ Battery for the Analysis of the Aphasic Deficits (BADA) } \\
\hline Object comprehension subtest & $40 / 40$ & $40 / 40$ & $38 / 40$ \\
\hline Action comprehension subtest & $18 / 20$ & $20 / 20$ & $20 / 20$ \\
\hline Object naming subtest & $25 / 30$ & $18 / 30$ & $4 / 30$ \\
\hline Action naming subtest & $21 / 28$ & $12 / 28$ & $1 / 28$ \\
\hline Sentence Comprehension & $58 / 60$ & $40 / 60$ & $44 / 60$ \\
\hline
\end{tabular}

Bold data indicate scores below normal cut-off.

These parameters are consistent with the safety recommendations for rTMS (Rossi, Hallett, Rossini, Pascual-Leone, \& Safety of TMS Consensus Group, 2009). The use of psychopharmacological agents that could interfere with the test performance or diagnosis and MRI evidence of relevant cerebrovascular changes unrelated to the main diagnosis of the patient were additional exclusion criteria.

\section{METHODS}

\section{TMS}

Patients were randomly assigned to one of two conditions: (1) Real-Real (RR), in which the patient received four weeks of rTMS stimulation of the left dorsolateral prefrontal cortex (DLPFC) combined with the speech therapy (P1); and (2) Placebo-Real (PR), in which patients received left DLPFC placebo stimulation combined with the speech therapy during the first two weeks followed by two weeks of real stimulation combined with speech therapy (P2 and P3). Each week of rTMS treatment consisted of 5 


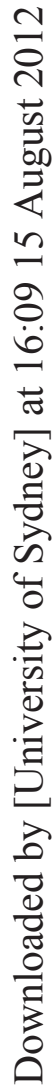

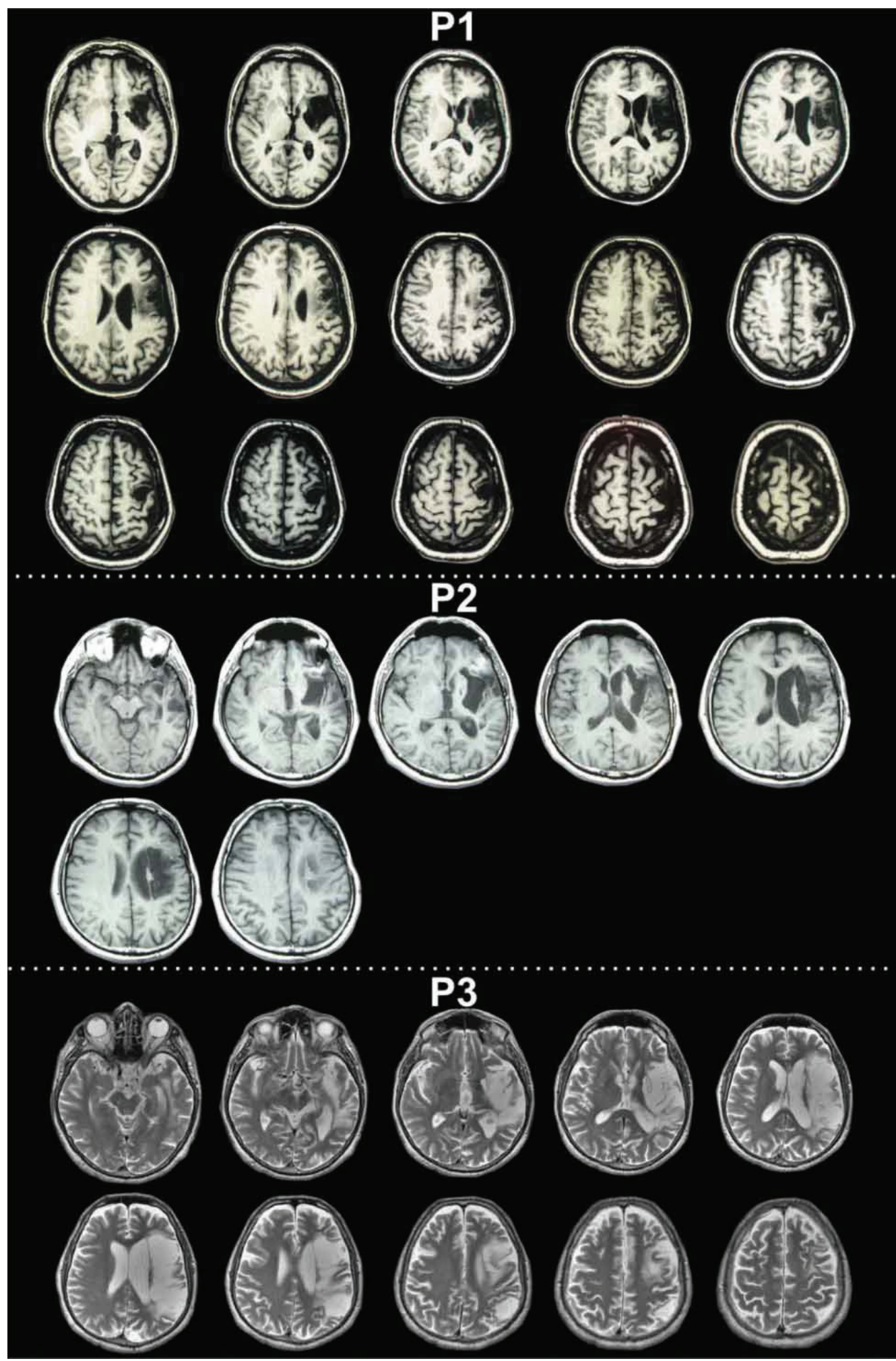

Figure 1. Structural MRIs for each patient. All the axial slices (thickness of $5 \mathrm{~mm}$ for P2 and P3 and of $4 \mathrm{~mm}$ for P1), where a lesion was present, are shown in a radiological convention (left side of brain is on the right). 
sessions that comprised a total of 50 minutes/day (25 minutes of rTMS plus 25 minutes of speech therapy). rTMS was delivered using a Magstim unit featuring a double $70 \mathrm{~mm}$ air-cooled coil. Before starting the rTMS treatment, the right motor cortex excitability stimulation threshold was established for each subject (mean $\pm S D: 54 \% \pm 10$ ). The stimulation intensity used during the experiment was set to $90 \%$ of each subject's motor threshold. Trains of high-frequency $(20 \mathrm{~Hz})$ rTMS were delivered in short periods $(2$ sec duration) separated by longer periods $(28 \mathrm{sec})$ of no stimulation, for each 25-minute daily rTMS session. The total number of pulses for each session was 2000 (40 stimuli/train, 50 trains). These parameters are consistent with safety recommendations for rTMS (Rossi et al., 2009). Furthermore, all participants tolerated rTMS well and did not report any adverse effects. In the placebo condition, a sham coil was used. We localised the target areas using the SofTaxic neuronavigator system (www.emsmedical.net) on an MRI template. Based on these estimated MRIs, the average location of the stimulating points was centred on Talairach coordinates $X=-35, Y=24$, $\mathrm{Z}=48$, corresponding to the left DLPFC (Brodmann Area 8/9). To stimulate the DLPFC, the coil was placed with the junction of the two coil wings above the target point. During the experiment, the coil was fixed with a mechanical support.

\section{Rationale for choosing the DLPFC as the rTMS target area}

High-frequency rTMS to the left DLPFC results in reduced action naming latencies in young subjects (Cappa, Sandrini, Rossini, Sosta, \& Miniussi, 2002), and in an increased number of correct responses for action naming in patients with mild Alzheimer's disease (AD), for both classes of stimuli (actions and objects) in moderate-to-severe AD patients (Cotelli et al., 2006; Cotelli, Manenti, Cappa, Zanetti, \& Miniussi, 2008). Moreover, in an off-line study, a significant effect of left DLPFC stimulation (25 minutes/ day for 5 days/week for 4 weeks) on language functioning, together with a lasting benefit at 6 months, was also found in an AD patient sample (Cotelli et al., 2011). Based on these observations, we hypothesised that high-frequency rTMS stimulation over the left DLPFC would result in improved naming in patients with post-stroke chronic aphasia. An additional hypothesis was that, combining this rTMS treatment with speech therapy, could enhance the improvement of the language deficit.

\section{BEHAVIOURAL ASSESSMENT}

Participants were assessed before therapy (baseline), after the first 2 weeks of treatment, and at the end of the 4 weeks of therapy. Tests including the following: a neuropsychological battery for reasoning and verbal fluency 
(Lezak, Howieson, \& Loring, 2004); the Aachener Aphasie Test (AAT; Luzzatti et al., 1994); the object and action naming subtests, comprehension and sentence comprehension subtests of the Battery for the Analysis of the Aphasic Deficit (BADA; Miceli, Laudanna, Burani, \& Capasso, 1994). See Table 2 for details.

\section{Stimuli selection}

To select stimuli for the therapy and to test for generalisation effects, all patients completed two oral naming tasks and one oral comprehension task. The oral naming task was repeated twice, on two consecutive days, to ensure a stable baseline before introducing therapy and to select the therapy items. Stimuli for the oral naming task were 340 black-and-white 2-dimensional line drawings representing objects, which were taken from the 795 corpus of objects and nouns of the CRL-IPNP (Center for Research in Language - International Picture Naming Project; http://crl.ucsd.edu) (Bates et al., 2000). These stimuli were normalised with healthy and brain damaged populations across seven international sites and languages; items were coded for a number of variables known to influence naming difficulty (e.g., word frequency, age of acquisition, degree of imageability, etc.). In total, 349 pictures of objects were displayed twice (on two consecutive days) on a computer screen using Presentation software v. 12.0 (neurobehavioural systems: http://www.neurobs.com), with each picture being presented for a maximum of 10 seconds. Each participant was asked to name each picture as accurately as possible, and oral responses were recorded.

In addition, because we aimed to focus the therapy on word production, all misnamed pictures were included in a comprehension task to make sure that the participants understood the word. In the comprehension task, participants were asked whether a picture presented for a maximum of 10 seconds corresponded or not to the spoken word. The participant was questioned about the picture's name during three consecutive trials, including the target's correct name, a semantic distractor and an unrelated distractor (e.g., for the picture of a bottle, the questions were "Is it a bottle?", "Is it a glass?" or "Is it a calendar?", respectively). The presentation of the items and the order of the distractors were randomised. Only items for which no errors were made in any of the three trials were selected for the rehabilitation list.

The rehabilitation list was further split into two sets: the "therapy" list, including the items to be treated; and the "control" list, with items not to be treated (untreated items). The two lists were balanced for a number of variables related to the participant's performance during the assessment of naming abilities; more specifically, these variables included the percent of correct picture naming across assessment sessions (one time or never across two sessions), target word frequency, number of syllables and number of 
letters, as well as semantic category (i.e., living or non-living). Furthermore, the two sets were split into two balanced sub-sets to be used during the first and the second week of treatment or to be used during the third and the fourth week, respectively (first and second vs. third and fourth).

Given the type of procedure used to select the "therapy" and "control" lists, each participant ended with a personalised set of items, which ensured the within- and across-subject validity of the design.

\section{Therapy protocol}

Participants underwent 25 minutes of speech therapy immediately following each rTMS treatment on a daily basis for 4 weeks. In general, the procedure included repetition and reading of the target word to facilitate naming, and an articulatory suppression task (average duration of articulatory suppression: 10 seconds) (Baddeley, Lewis, \& Vallar, 1984). For every error, the trial was repeated again (at the end of the list). The patient was seated in front of a computer screen in a quiet room while the therapy protocol was displayed using Presentation software v. 12.0 (neurobehavioural systems: http://www.neurobs.com). The pictures showing the items to be treated and the written words used during the treatment were presented on the computer screen. The treatment involved several steps to elicit the production of a target noun, specifically:

Step 1: Repetition of the target word (the target word was spoken by the therapist and the participant was asked to repeat it three times).

Articulatory suppression task: Interference with articulatory codes caused by the uttering of an irrelevant speech sound (i.e., bla, bla, bla).

In the suppression condition, participants received instructions to start uttering the syllable "bla". The suppression was carried on during the interval between step 1 and step 2 and between step 3 and step 4 .

Step 2: Oral picture naming (the target picture was presented on the computer screen and the participant was asked to retrieve its correct name).

Step 3: Reading of the target word (the target written word was presented on the computer screen and the participant was asked to read it).

Articulatory suppression task: Interference with articulatory codes caused by the uttering of an irrelevant speech sound (i.e., bla, bla, bla).

Step 4: Oral picture naming (the target picture was presented on the computer screen and the participant was asked to retrieve its correct name, and say it aloud).

The complete procedure was repeated when at least one of the two requested naming responses was incorrect.

At the end of the therapy, object naming was reassessed, with their respective lists prepared during the pre-therapy assessment. At the end of the first two weeks of treatment, an equal number of treated and untreated items corresponding to the first two weeks were tested, whereas at the end of the 
treatment and at follow-up visits, all treated and untreated items corresponding to the four weeks of the experiment were tested.

\section{RESULTS}

Significant improvements were found for object naming in the experimental stimuli set. For each participant, we calculated the baseline for the therapy and control lists. The percentage correct at baseline for each participant corresponded to the number of items correctly named in one of the two naming assessment sessions, divided by two and further divided by the total number of items in the therapy and control lists (both the therapy and control lists included the same number of items) multiplied by 100. For each PWA, a $\chi^{2}$ comparison, with Yates correction, was applied to compare performance scores after 2, 4, 12, 24 and 48 weeks, with respect to baseline and for both the treated and untreated items. In contrast, no changes were recorded in the standard neuropsychological assessment including formal language assessment (AAT and BADA) after the therapy.

For treated items, the three PWAs showed improvement after 2 weeks of combined rTMS-behavioural therapy as compared to baseline, and this significant improvement persisted at 4, 12, 24 and 48 weeks. Moreover, the improvement was also significant with untreated items after 2 weeks of combined rTMS and behavioural therapy and at weeks 4, 12, 24 and 48 (see Figure 2 and Table 3 for details).

Additionally, in order to examine the generalisation of effects to untreated items, we compared the accuracy scores for trained and untrained items for each PWA and for each time-point using Yates-corrected $\chi^{2}$ comparisons. The results showed a significant effect on treated items (vs. untreated items) at the 2- and 4-week post-treatment measurement points, in all three cases, whereas the difference between naming treated and untreated images decreased over time, and, in most cases, vanished at follow-up assessments beginning at 12 weeks post-entry/baseline in two of the three cases (see Table 3 for details).

\section{DISCUSSION}

Our preliminary findings provide additional evidence for the beneficial effects of brain stimulation in combination with targeted behavioural training in PWAs suffering from anomia. In particular, a long-lasting effect of combined rTMS and behavioural therapy was observed; this effect was still present at 48 weeks after the beginning of the combined rTMS-speech therapy intervention on the therapy list, but not on the standardised tests. This result is consistent with previous reports of enhanced cognitive performance following noninvasive brain stimulation (i.e., rTMS or tDCS) to specific cortical areas in 
patients with a variety of neurological diseases (Baker et al., 2010; Berthier, \& Pulvermuller, 2011; Cotelli et al., 2006, 2008; Fiori et al., 2011; Martin et al., 2004; Monti et al., 2008; Naeser, Martin, Nicholas, Baker, Seekins,
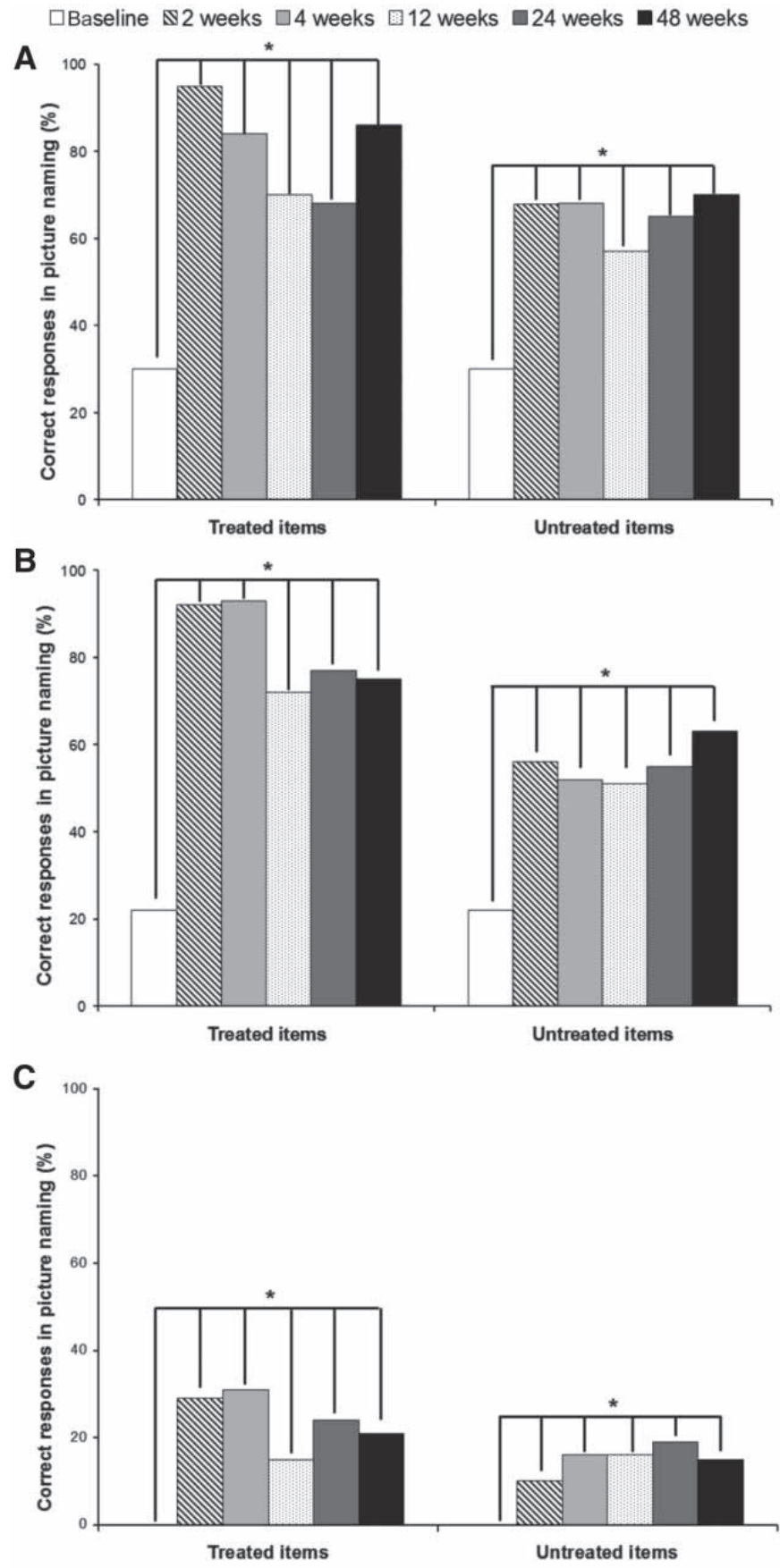

Figure 2. Percentage of correct responses in the picture-naming task for treated and untreated items at baseline and 2, 4, 12, 24 and 48 weeks after the beginning of treatment for patients P1 (A), P2 (B) and P3 (C). ${ }^{*} p<.05$. 


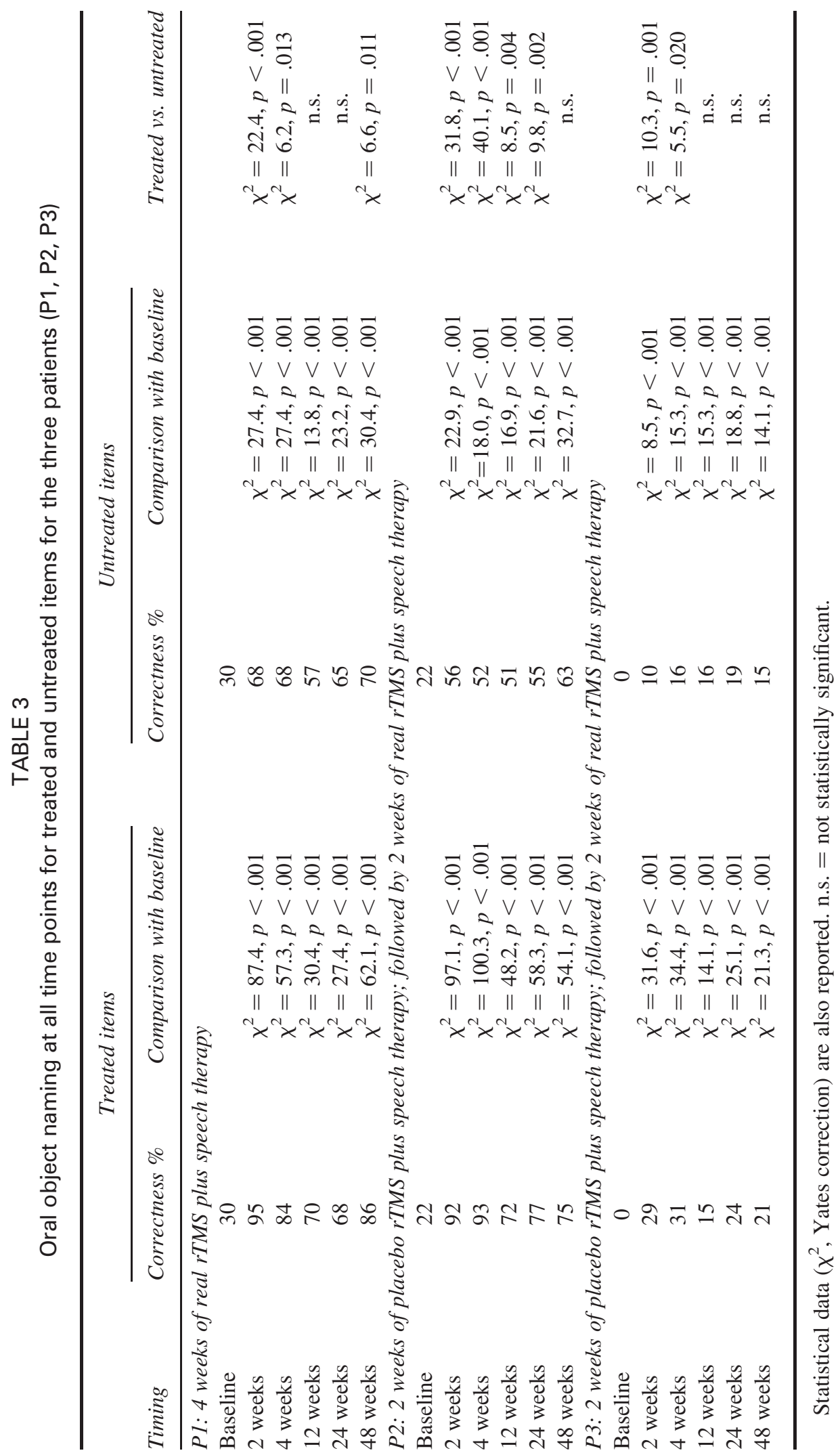


Helm-Estabrooks, et al., 2005; Naeser, Martin, Nicholas, Baker, Seekins, Kobayashi, et al., 2005). Moreover, the results of our study are also consistent with previous evidence regarding the increased efficacy of daily combined rTMS or tDCS plus cognitive rehabilitation (Baker et al., 2010; Fiori et al., 2011; Floel et al., 2011; Fridriksson et al., 2011; Kakuda, Abo, Uruma, et al., 2010; Kang et al., 2011; Martin, Naeser, Ho, Treglia, et al., 2009; Naeser, Martin, Treglia, et al., 2010; Weiduschat et al., 2011).

The present study provides additional evidence supporting the use of combined behavioural and brain stimulation approaches to achieve successful outcomes in aphasia therapy. The heterogeneity of the approaches used to date makes comparison of rTMS studies complex, i.e., (1) low-frequency rTMS over the unaffected hemisphere to suppress the presence of over-activation in the right frontal area due, in part, to lack of transcallosal inhibition from the damaged left frontal area; and (2) high-frequency rTMS over the damaged hemisphere aiming at facilitating spared regions surrounding the damaged areas.

Regarding tDCS, both anodal and cathodal stimulation applied over the lesional hemisphere improved language in post-stroke aphasia patients. What are the possible mechanisms responsible for these effects? It has been hypothesised that both tDCS and rTMS can affect the cortical plastic changes following stroke in a positive way and that these effects may outlast the stimulation period (Ridding \& Rothwell, 2007).

Several studies support the idea that a favourable recovery from post-stroke aphasia is associated with a predominant reactivation of ipsilesional areas (Heiss, Emunds, \& Herholz, 1993; Thiel et al., 1998, 2001; Winhuisen et al., 2005, 2007). Recent reviews highlight that several recruitment mechanisms may occur, including persistent function in spared areas, compensatory recruitment of alternate nodes, and involvement of areas that may hinder recovery (Turkeltaub, Messing, Norise, \& Hamilton, 2011). Based on these ideas, we applied high-frequency rTMS over the left DLPFC to increase cortical excitability and our results are consistent with this perspective. No adverse effects have been reported, supporting the safety of this approach (Rossi et al., 2009).

These facilitation effects may be related to changes in cortical excitability and plasticity (Ridding \& Rothwell, 2007). One possible explanation for these stimulation effects is that they may be mediated by the enhancement of compensatory modifications in functional networks associated with a specific function (Fridriksson, 2010). These modifications of cortical activity through the use of stimulation may readjust the pathological patterns of brain activity, thus providing an opportunity to normalise activity patterns within the affected functional networks (Thut \& Miniussi, 2009).

The present findings suggest that rTMS-induced modulation of short- and/ or long-range cortical synaptic efficacy and connectivity, which potentiates 
the system within the language network, leads to increased effects of speech therapy (see Miniussi \& Rossini, this issue).

The major limitations of this preliminary study were the small number of patients and the lack of a placebo stimulation group, for 2 and 4 weeks, with and without speech therapy. Thus, the main findings of this study, i.e., that combined behavioural-rTMS treatment induced a long-lasting effect on treated items (up to 48 weeks), and contributed to a generalisation of therapy effects to untreated items (observed earlier post-treatment in all three cases, but not always observed later) (Miceli, Amitrano, Capasso, \& Caramazza, 1996), need to be confirmed using a larger sample. The inclusion of a control group receiving only language therapy is required to separate the respective contribution of language therapy alone, and in combination with real or placebo rTMS treatments.

\section{REFERENCES}

Antal, A., Nitsche, M. A., Kruse, W., Kincses, T. Z., Hoffmann, K. P., \& Paulus, W. (2004). Direct current stimulation over V5 enhances visuomotor coordination by improving motion perception in humans. Journal of Cognitive Neuroscience, 16, 521-527.

Baddeley, A. D., Lewis, V., \& Vallar, G. (1984). Exploring the articulatory loop. Quarterly Journal of Experimental Psychology, 36, 233-252.

Baker, J. M., Rorden, C., \& Fridriksson, J. (2010). Using transcranial direct-current stimulation to treat stroke patients with aphasia. Stroke, 41, 1229-1236.

Barwood, C. H., Murdoch, B. E., Whelan, B. M., Lloyd, D., Riek, S., O'Sullivan, J., Coulthard, A., \& Wong, A. (2011). Improved language performance subsequent to low-frequency rTMS in patients with chronic non-fluent aphasia post-stroke. European Journal of Neurology, 18, 935-943.

Barwood, C. H., Murdoch, B. E., Whelan, B. M., Lloyd, D., Riek, S., O'Sullivan, J., Coulthard, A., Wong, A., Aitken, P., \& Hall, G. (2011). The effects of low frequency Repetitive Transcranial Magnetic Stimulation (rTMS) and sham condition rTMS on behavioural language in chronic non-fluent aphasia: Short term outcomes. NeuroRehabilitation, 28, 113-128.

Bates, E., Andonova, E., D’ Amico, S., Jacobsen, T., Kohnert, K., Lu, C. C., et al. (2000). Introducing the CRL International Picture-Naming Project (CRL-IPNP). Center for Research in Language Newsletter La Jolla: University of California San Diego, 12.

Berthier, M. L., \& Pulvermuller, F. (2011). Neuroscience insights improve neurorehabilitation of poststroke aphasia. Nature Reviews Neurology, 7, 86-97.

Bhogal, S. K., Teasell, R., \& Speechley, M. (2003). Intensity of aphasia therapy, impact on recovery. Stroke, 34, 987-993.

Bindman, L. J., Lippold, O. C., \& Redfearn, J. W. (1962). Long-lasting changes in the level of the electrical activity of the cerebral cortex produced bypolarizing currents. Nature, 196, $584-585$.

Black-Schaffer, R. M., \& Osberg, J. S. (1990). Return to work after stroke: Development of a predictive model. Archives of Physical Medicine and Rehabilitation, 71, 285-290.

Cappa, S. F., Benke, T., Clarke, S., Rossi, B., Stemmer, B., \& van Heugten, C. M. (2005). EFNS guidelines on cognitive rehabilitation: Report of an EFNS task force. European Journal of Neurology, 12, 665-680. 
Cappa, S. F., Benke, T., Clarke, S., Rossi, B., Stemmer, B., van Heugten, C. M., \& European Federation of Neurological Societies (2003). EFNS guidelines on cognitive rehabilitation: Report of an EFNS task force. European Journal of Neurology, 10, 11-23.

Cappa, S. F., Sandrini, M., Rossini, P. M., Sosta, K., \& Miniussi, C. (2002). The role of the left frontal lobe in action naming: rTMS evidence. Neurology, 59, 720-723.

Cotelli, M., Calabria, M., Manenti, R., Rosini, S., Zanetti, O., Cappa, S. F., et al. (2011). Improved language performance in Alzheimer disease following brain stimulation. Journal of Neurology, Neurosurgery, \& Psychiatry, 82, 794-797.

Cotelli, M., Manenti, R., Cappa, S. F., Geroldi, C., Zanetti, O., Rossini, P. M., et al. (2006). Effect of transcranial magnetic stimulation on action naming in patients with Alzheimer disease. Archives of Neurology, 63, 1602-1604.

Cotelli, M., Manenti, R., Cappa, S. F., Zanetti, O., \& Miniussi, C. (2008). Transcranial magnetic stimulation improves naming in Alzheimer disease patients at different stages of cognitive decline. European Journal of Neurology, 15, 1286-1292.

Engelter, S. (2006). Aphasia in stroke patients: Frequency and significance. Praxis, 95, 489-492.

Fertonani, A., Rosini, S., Cotelli, M., Rossini, P. M., \& Miniussi, C. (2010). Naming facilitation induced by transcranial direct current stimulation. Behavioural Brain Research, 208, 311-318.

Fiori, V., Coccia, M., Marinelli, C. V., Vecchi, V., Bonifazi, S., Ceravolo, M. G., et al. (2011). Transcranial direct current stimulation improves word retrieval in healthy and nonfluent aphasic subjects. Journal of Cognitive Neuroscience, 23, 2309-2323.

Floel, A., Meinzer, M., Kirstein, R., Nijhof, S., Deppe, M., Knecht, S., et al. (2011). Short-term anomia training and electrical brain stimulation. Stroke, 42, 2065-2067.

Fregni, F., Boggio, P. S., Nitsche, M., Bermpohl, F., Antal, A., Feredoes, E., et al. (2005). Anodal transcranial direct current stimulation of prefrontal cortex enhances working memory. Experimental Brain Research, 166, 23-30.

Fridriksson, J. (2010). Preservation and modulation of specific left hemisphere regions is vital for treated recovery from anomia in stroke. Journal of Neuroscience, 30, 11558-11564.

Fridriksson, J., Richardson, J. D., Baker, J. M., \& Rorden, C. (2011). Transcranial direct current stimulation improves naming reaction time in fluent aphasia: A double-blind, sham-controlled study. Stroke, 42, 819-821.

Galletta, E. E., Rao, P. R., \& Barrett, A. M. (2011). Transcranial magnetic stimulation (TMS): Potential progress for language improvement in aphasia. Topics in Stroke Rehabilitation, 18, 87-91.

Hamilton, R. H., Sanders, L., Benson, J., Faseyitan, O., Norise, C., Naeser, M., et al. (2010). Stimulating conversation: Enhancement of elicited propositional speech in a patient with chronic non-fluent aphasia following transcranial magnetic stimulation. Brain \& Language, $113,45-50$.

Heiss, W. D., Emunds, H. G., \& Herholz, K. (1993). Cerebral glucose metabolism as a predictor of rehabilitation after ischemic stroke. Stroke, 24, 1784-1788.

Iyer, M. B., Mattu, U., Grafman, J., Lomarev, M., Sato, S., \& Wassermann, E. M. (2005). Safety and cognitive effect of frontal DC brain polarization in healthy individuals. Neurology, 64, 872-875.

Jung, T. D., Kim, J. Y., Lee, Y. S., Kim, D. H., Lee, J. J., Seo, J. H., et al. (2010). Effect of repetitive transcranial magnetic stimulation in a patient with chronic crossed aphasia: fMRI study. Journal of Rehabilitation Medicine, 42, 973-978.

Kakuda, W., Abo, M., Kaito, N., Watanabe, M., \& Senoo, A. (2010). Functional MRI-based therapeutic rTMS strategy for aphasic stroke patients: A case series pilot study. International. Journal of Neuroscience, 120, 60-66. 
Kakuda, W., Abo, M., Uruma, G., Kaito, N., \& Watanabe, M. (2010). Low-frequency rTMS with language therapy over a 3-month period for sensory-dominant aphasia: Case series of two post-stroke Japanese patients. Brain Injury, 24, 1113-1117.

Kang, E. K., Kim, Y. K., Sohn, H. M., Cohen, L. G., \& Paik, N. J. (2011). Improved picture naming in aphasia patients treated with cathodal tDCS to inhibit the right Broca's homologue area. Restorative Neurology and Neuroscience, 29, 141-152.

Kelly, H., Brady, M. C., \& Enderby, P. (2010). Speech and language therapy for aphasia following stroke. Cochrane Database Systematic Reviews, 5, CD000425.

Laska, A. C., Hellblom, A., Murray, V., Kahan, T., \& Von Arbin, M. (2001). Aphasia in acute stroke and relation to outcome. Journal of Internal Medicine, 249, 413-422.

Lezak, M., Howieson, D., \& Loring, D. W. (2004). Neuropsychological assessment (4th ed.). Oxford, UK: Oxford University Press.

Liebetanz, D., Nitsche, M. A., Tergau, F., \& Paulus, W. (2002). Pharmacological approach to the mechanisms of transcranial DC-stimulation-induced after-effects of human motor cortex excitability. Brain, 125, 2238-2247.

Luzzatti, C., Willmes, K., De Bleser, R., Bianchi, A., Chiesa, G., De Tanti, A., et al. (1994). Nuovi dati normativi per la versione italiana dell'Aachener Aphasia test. Archivio di Psicologia, Neurologia e Psichiatria, 55, 1086-1131.

Martin, P. I., Naeser, M. A., Ho, M., Doron, K. W., Kurland, J., Kaplan, J., et al. (2009). Overt naming fMRI pre- and post-TMS: Two nonfluent aphasia patients, with and without improved naming post-TMS. Brain \& Language, 111, 20-35.

Martin, P. I., Naeser, M. A., Ho, M., Treglia, E., Kaplan, E., Baker, E. H., et al. (2009). Research with transcranial magnetic stimulation in the treatment of aphasia. Current Neurology and Neuroscience Reports, 9, 451-458.

Martin, P. I., Naeser, M. A., Theoret, H., Tormos, J. M., Nicholas, M., Kurland, J., et al. (2004). Transcranial magnetic stimulation as a complementary treatment for aphasia. Seminars in Speech and Language, 25, 181-191.

Miceli, G., Amitrano, A., Capasso, R., \& Caramazza, A. (1996). The treatment of anomia resulting from output lexical damage: Analysis of two cases. Brain, \& Language, 52, 150-174.

Miceli, G., Laudanna, A., Burani, C., \& Capasso, R. (1994). Batteria per l'Analisi dei Deficit Afasici. B.A.D.A. (Battery for Analysis of Aphasic Deficits). Milano: CEPSAG, Università Cattolica del Sacro Cuore.

Miniussi, C., Cappa, S. F., Cohen, L. G., Floel, A., Fregni, F., Nitsche, M. A., et al. (2008). Efficacy of repetitive transcranial magnetic stimulation/transcranial direct current stimulation in cognitive neurorehabilitation. Brain Stimulation, 1, 326-336.

Monti, A., Cogiamanian, F., Marceglia, S., Ferrucci, R., Mameli, F., Mrakic-Sposta, S., et al. (2008). Improved naming after transcranial direct current stimulation in aphasia. Journal of Neurology, Neurosurgery, \& Psychiatry, 79, 451-453.

Naeser, M. A., Martin, P. I., Lundgren, K., Klein, R., Kaplan, J., Treglia, E., et al. (2010). Improved language in a chronic nonfluent aphasia patient after treatment with CPAP and TMS. Cognitive and Behavioral Neurology, 23, 29-38.

Naeser, M. A., Martin, P. I., Nicholas, M., Baker, E. H., Seekins, H., Helm-Estabrooks, N., et al. (2005). Improved naming after TMS treatments in a chronic, global aphasia patient - case report. Neurocase, 11, 182-193.

Naeser, M. A., Martin, P. I., Nicholas, M., Baker, E. H., Seekins, H., Kobayashi, M., et al. (2005). Improved picture naming in chronic aphasia after TMS to part of right Broca's area: An open-protocol study. Brain \& Language, 93, 95-105.

Naeser, M. A., Martin, P. I., Treglia, E., Ho, M., Kaplan, E., Bashir, S., et al. (2010). Research with rTMS in the treatment of aphasia. Restorative Neurology and Neuroscience, 28, 511-529. 
Nitsche, M. A., Schauenburg, A., Lang, N., Liebetanz, D., Exner, C., Paulus, W., et al. (2003). Facilitation of implicit motor learning by weak transcranial direct current stimulation of the primary motor cortex in the human. Journal of Cognitive Neuroscience, 15, 619-626.

Ohn, S. H., Park, C. I., Yoo, W. K., Ko, M. H., Choi, K. P., Kim, G. M., et al. (2008). Timedependent effect of transcranial direct current stimulation on the enhancement of working memory. Neuroreport, 19, 43-47.

Paolucci, S., Antonucci, G., Pratesi, L., Traballesi, M., Lubich, S., \& Grasso, M. G. (1998). Functional outcome in stroke inpatient rehabilitation: Predicting no, low and high response patients. Cerebrovascular Diseases, 8, 228-234.

Reis, J., Schambra, H. M., Cohen, L. G., Buch, E. R., Fritsch, B., Zarahn, E., et al. (2009). Noninvasive cortical stimulation enhances motor skill acquisition over multiple days through an effect on consolidation. Proceedings of the National Academy of Sciences, 106, 1590-1595.

Ridding, M. C., \& Rothwell, J. C. (2007). Is there a future for therapeutic use of transcranial magnetic stimulation? Nature Reviews Neuroscience, 8, 559-567.

Rossi, S., Hallett, M., Rossini, P. M., Pascual-Leone, A., \& Safety of TMS Consensus Group (2009). Safety, ethical considerations, and application guidelines for the use of transcranial magnetic stimulation in clinical practice and research. Clinical Neurophysiology, 120, 2008-2039.

Sparing, R., Dafotakis, M., Meister, I. G., Thirugnanasambandam, N., \& Fink, G. R. (2008). Enhancing language performance with non-invasive brain stimulation - a transcranial direct current stimulation study in healthy humans. Neuropsychologia, 46, 261-268.

Szaflarski, J. P., Vannest, J., Wu, S. W., DiFrancesco, M. W., Banks, C., \& Gilbert, D. L. (2011). Excitatory repetitive transcranial magnetic stimulation induces improvements in chronic post-stroke aphasia. Medical Science Monitor, 17, 132-139.

Thiel, A., Herholz, K., Koyuncu, A., Ghaemi, M., Kracht, L. W., Habedank, B., et al. (2001). Plasticity of language networks in patients with brain tumors: A positron emission tomography activation study. Annals of Neurology, 50, 620-629.

Thiel, A., Herholz, K., von Stockhausen, H. M., van Leyen-Pilgram, K., Pietrzyk, U., Kessler, J., et al. (1998). Localization of language-related cortex with 15O-labeled water PET in patients with gliomas. NeuroImage, 7, 284-295.

Thut, G., \& Miniussi, C. (2009). New insights into rhythmic brain activity from TMS-EEG studies. Trends in Cognitive Sciences, 13, 182-189.

Tilling, K., Sterne, J. A., Rudd, A. G., Glass, T. A., Wityk, R. J., \& Wolfe, C. D. (2001). A new method for predicting recovery after stroke. Stroke, 32, 2867-2873.

Turkeltaub, P. E., Messing, S., Norise, C., \& Hamilton, R. H. (2011). Are networks for residual language function and recovery consistent across aphasic patients? Neurology, 76, 1726-1734.

Vines, B. W., Schnider, N. M., \& Schlaug, G. (2006). Testing for causality with transcranial direct current stimulation: Pitch memory and the left supramarginal gyrus. Neuroreport, 17, 1047-1050.

Weiduschat, N., Thiel, A., Rubi-Fessen, I., Hartmann, A., Kessler, J., Merl, P., et al. (2011). Effects of repetitive transcranial magnetic stimulation in aphasic stroke: A randomized controlled pilot study. Stroke, 42, 409-415.

Winhuisen, L., Thiel, A., Schumacher, B., Kessler, J., Rudolf, J., Haupt, W. F., et al. (2005). Role of the contralateral inferior frontal gyrus in recovery of language function in poststroke aphasia: A combined repetitive transcranial magnetic stimulation and positron emission tomography study. Stroke, 36, 1759-1763.

Winhuisen, L., Thiel, A., Schumacher, B., Kessler, J., Rudolf, J., Haupt, W. F., et al. (2007). The right inferior frontal gyrus and poststroke aphasia: A follow-up investigation. Stroke, 38, $1286-1292$.

You, D. S., Kim, D. Y., Chun, M. H., Jung, S. E., \& Park, S. J. (2011). Cathodal transcranial direct current stimulation of the right Wernicke's area improves comprehension in subacute stroke patients. Brain \& Language, 119, 1-5. 